\title{
Technology watch
}

\section{TAKE YOUR PICh}

It has been notoriously difficult to analyse the protein composition of defined chromatin loci. Déjardin and Kingston have now developed a strategy, named PICh (proteomics of isolated chromatin segments), that uses nucleic acid hybridization to purify an endogenous chromatin locus in sufficient quantities. The associated proteins are subsequently determined by mass spectrometry (MS). Solubilized chromatin from fixed cells is hybridized to a specific probe, and locked nucleic acid-containing oligonucleotides help to increase the stability of the probe-chromatin interactions. The hybridized chromatin is then captured on magnetic beads, the hybrids are eluted and the chromatin-bound proteins are crosslinked with formaldehyde prior to MS analysis. The authors used telomeric chromatin to validate the technology. PICh identified $\sim 85 \%$ of the known components at two telomere types - one is maintained by telomerase and one uses the telomerase-independent ALT (alternative lengthening of telomeres) pathway. In addition, out of the eight novel telomere-associated proteins identified by PICh that were tested, seven show clear, and one shows likely, association with the locus, as determined by fluorescence microscopy. The unexpected association of ALT telomeres with certain orphan receptors, which are known transcription factors, might underlie an additional function in ALT maintenance. PICh also provides insight into the relative abundance of chromatin-associated proteins, including histones, which might have important functional implications.

ORIGINAL RESEARCH PAPER Déjardin, J. \& Kingston, R. E. Purification of proteins associated with specific genomic loci. Cell 136, 175-186 (2009)

\section{SUMO GOES GLOBAL}

To analyse the full range of small ubiquitin-related modifier (SUMO) functions, Makhnevych et al. mapped the physical and functional interactions of SUMO. Using affinity purification combined with mass spectrometry, they identified 232 polypeptides from Saccharomyces cerevisiae cell lysate that interacted specifically with recombinant SUMO protein. A yeast two-hybrid screen using SUMO protein as bait identified 34 putative SUMO interactors, of which several were already known. More than 500 genes and $\sim 1,400$ synthetic genetic interactions were mapped by synthetic genetic array (SGA) analysis using eight different query genes. Analysis of the combined physical and genetic interactions allowed the authors to assign $93 \%$ of the SUMO pathway to 15 different biological processes. The integrated data set, together with previously published SUMO conjugate data sets, has already proven to be an extremely valuable resource, given the identification of roles for the SUMO pathway in the function of the $\mathrm{AAA}^{+}$ATPase $\mathrm{Cdc} 48$, the regulation of lipid metabolism, localization of the ATP-dependent endonuclease Dna2 and recovery from the DNA-damage checkpoint.

ORIGINAL RESEARCH PAPER Makhnevych, T. et al. Global map of SUMO function function revealed by protein-protein interaction and genetic networks. Mol. Cell 33 , 124-135 (2009) 